\title{
A Reliable Based Multicast Approach Using by Fuzzy Logic System for Improvement QoS in Mobile Ad-hoc Networks
}

\author{
Shahram Zandiyan ${ }^{1}$, Abbas Mirzaei Somarin ${ }^{2}$ \\ ${ }^{1}$ Department of Computer engineering. Ardabil Science and Research Branch. Islamic Azad University. \\ Ardabil, Iran. \\ ${ }^{2}$ Department of Computer engineering. Ardabil branch. Islamic Azad University. Ardabil, Iran. \\ * Corresponding author. Tel.: 00989141531130; email: A.mirzaei@iauardabil.ac.ir \\ Manuscript submitted September 10, 2017; accepted November 8, 2017. \\ doi: $10.17706 /$ jcp.13.7.862-875
}

\begin{abstract}
In this paper, we proposed a fuzzy logic based multicast approach to select two comparably stable routes by computing dynamic route lifetime in mobile ad-hoc networks (MANETs). The fuzzy logic weighted multi-criteria of the protocol is used to dynamically evaluate the active route lifetime in order to determine the appropriate routes. Multiple routing paths are established to provide multicast transmission and enhance the robust transmission against unreliability and limited bandwidth of wireless links. Due to frequent node movements, the topologies of mobile ad hoc networks change rapidly. The evaluation of dynamic route lifetime is challenging in the wireless networks. The fuzzy weighted logic multi-criteria are based on the minimum consumption energy of the nodes on the routes, the number of hop-counts and the controlled packages. The proposed approach, called RM-Approach (Reliable Multicast Approach) employ FLS (Fuzzy Logic System) to improvement QoS in MANETs. RM-Approach is evaluated through extensive simulations in the ns-2 environment. The results show that RM-Approach outperforms other existing solutions in terms of delivery ratio, end-to-end delay and lifetime.
\end{abstract}

Key words: MANETs, multicast routing protocol, fuzzy logic system, RM-approach.

\section{Introduction}

Dissemination of wireless devices with mobile networks became contemporary in this era. This led to the concern where Mobile Ad hoc Networks (MANETs) are premeditated and probed [1]-[4]. MANET conformities Infrastructure less networks, by exerting every node in the process of a router Utilization of Routing scheme and routing protocols encodes the efficiency of MANET. Arbitrary movement of the nodes and the radio propagation conditions are observed in MANET. In MANET, data are coded from source to copious destinations through multicast routing protocols in a coordinated multicast group community. Robustness is maintained by identifying the path failures and flexibility to the malicious attackers are the matter of contention which needs to be certified. Multicasting decisively reinforces applications in sample that are typified by proximity in concert. Mobility nature of the nodes causes path and link failure appended with the ensuing growth of malicious intruders thereby whole network connectivity gets ruined. Hence multicast routing protocol requires optimization to establish a staunch and flexible network against failures and attackers. In a typical network, the most crucial node and link failure origin remains problematic and erratic. The soundness in a node and link is figured through mobility model. Additionally the transmission competence is impacted by throughput in MANET. Moreover congestion of wireless network induces the capacity to be limited in MANET, consequently affecting the network reliability. This paper employs Fuzzy 
Logic technique [5]-[7]. to design an AODV based multicast protocol, which is improvement QoS. RM-Approach (Reliable Multicast Approach) is implemented in the NS-2 simulator to evaluate its performance. Rest of this paper is organized as follows. Section 2 presents the preliminaries of the research. In Section 3, we present the related works around the multicast approach and Section 4 brings details of the RM-Approach. Performance evaluation parameters and simulation results are introduced in Section 5. Finally, we present our conclusions in Section 6.

\section{Preliminaries}

In this section, we provide a brief review over some basic issues such as the multicast routing and the fuzzy logic system.

\subsection{Multicast Routing Protocol}

Multicast routing protocols play a considerable role in providing effective multicast infrastructures and in expand new applications. The multicast development started with the creation of Multicast backbone [8] and the corresponding Multicast routing protocol. Initial efforts were done in the classification and deployment of multicast protocols for a single flat topology. These protocols are categorized as intra domain protocols. Later on, the multicast community realized the need for developing so called inter domain routing protocols based on a hierarchical routing structure, as is the Internet. Multicast routing protocol extension has some limitations that illustrate why the protocols have not been widely perform since their initial design. One of the difficulties is that multicast needs to be engaged in a heterogeneous network with the size of the Internet. This is a difficult task, as there are a large the number of devices that need to be additionally configured. Another deployment problem is that network layer multicast, especially on inter domain level has been observed to be a hard task [9]. Many types of multicast routing protocols is developed, Some of which have become more popular and more Widely deployed on the Internet than others. The usage of a special protocol depends on the environment and the demand for different applications that use multicast technology. Although, a good comprehension of the protocols and of how they work is needed in order to evaluate the performance of these protocols in a real environment. In general, characterize protocols generates also understanding of the performance of the multicast technology. Fig. 1. shows that an example multicast routing protocols in mobile ad-hoc networks.

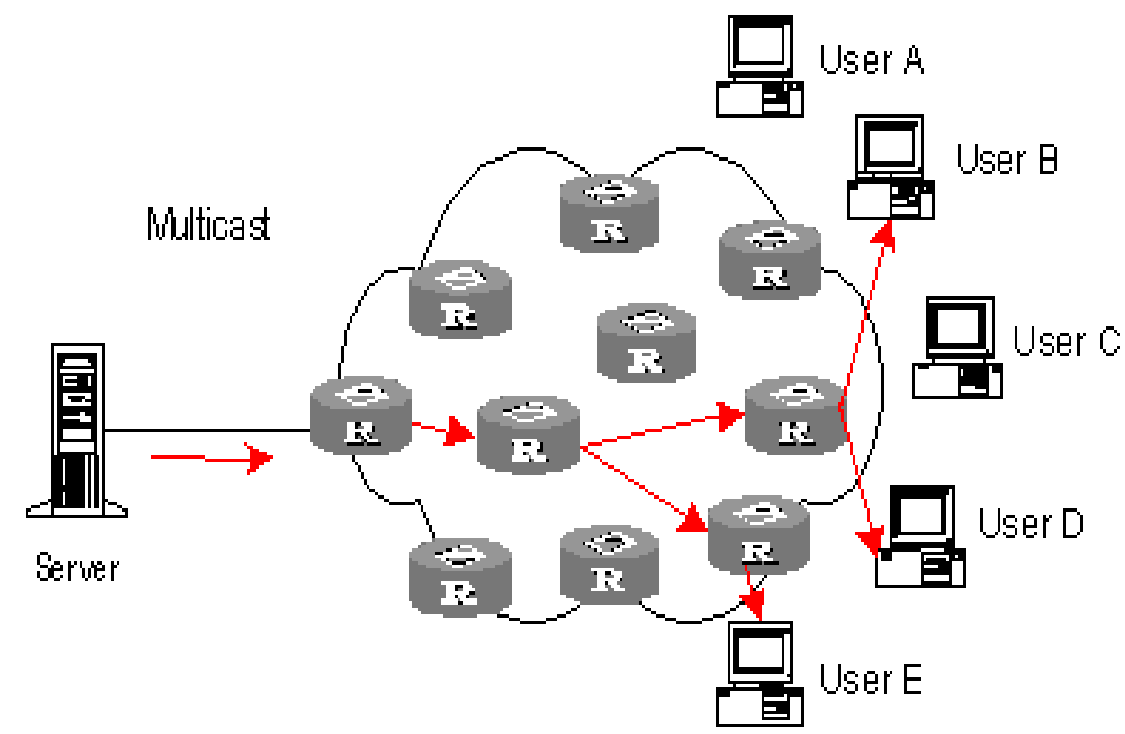

Fig. 1. Multicast routing protocols in ad hoc networks. 


\subsection{Fuzzy Logic System}

Fuzzy logic is a multi-valued logic in which the values of variables may be any real number between true (1) and false (0). Using fuzzy logic, an input space can be mapped into an output space. Fuzzy logic is used in situations where the available information is in form of partial truth that makes decision process very complicated. When it is difficult to identify an element whether it belongs to one set or another, fuzzy logic is the best option for making such decisions. Fuzzy logic is based on the fuzzy set theory. In Crisp Set theory, an element is either present or is not present in a set. On the other hand, fuzzy set theory deals with the degree of membership of an element in a particular set. An element may be the member of multiple sets at the same time. An element may partially be the member of set A and partially the member of set B. This implies that fuzzy set theory deals with the absence or presence of an element in a particular set. In addition it deals with the partial presence or partial absence of an element in a set as well. Membership function is used to define how each point in the input space is mapped to a membership value between 0 and 1 . The input to the membership function is a crisp value and output is a fuzzy value between 0 and 1 that shows the degree of membership in a set. Fuzzy logic uses different logical operators to carry out its operations, such as AND, OR, and NOT. AND operation is equivalent to min (minimum) operation, OR operation can find the maximum of given inputs, and NOT is the complement operator. Fuzzy sets and fuzzy operations are combined to create if-then rules.

Fuzzy logic is composed of these if-then rules. The if-part of the rule is called the antecedent while then-part is called the consequent [10]. A fuzzy logic system maps crisps inputs to crisp outputs. There are four components in a fuzzy logic system, namely rules, fuzzifier, inference mechanism, and defuzzifier as shown in Fig. 2. Rules are basically if-then rules which must be evaluated during an input/output process. The output of the system depends on these rules. If-then rules are designed by the experts of a particular field. Fuzzifier is responsible to take crisp numbers as input and give fuzzy sets as output. The activation of rules is dependent on the output of fuzzifier. Inference mechanism maps fuzzy sets into fuzzy sets. Inference mechanism is the decision making part of fuzzy logic system. Defuzzifier maps the fuzzy output of inference mechanism into crisp numbers to make it usable for further processing by the system [10].

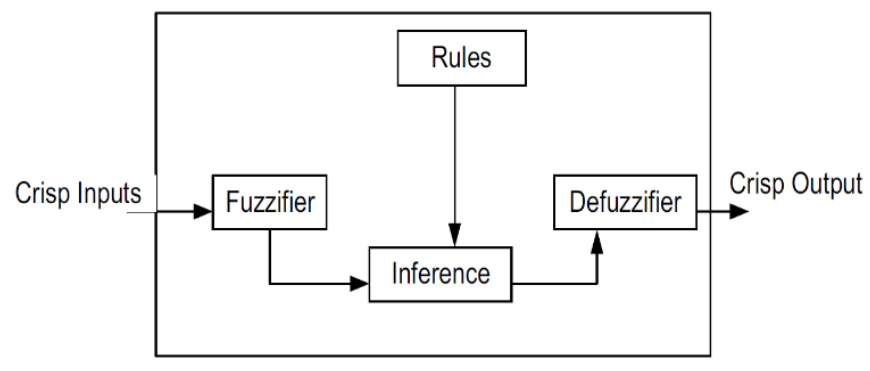

Fig. 2. Fuzzy logic system.

\section{Related Works}

Multicast routing has received considerable attentions in the literature.In paper [11], fuzzy cost based Multi Constrained Quality of Service Routing was proposed for optimal path selection based on bandwidth, end to end delay and the intermediate hops count. The path with maximum lifetime and minimum fuzzy cost was considered for best transmission. There was no stability of link present in this work. Lifetime of the network depends on link and node stability.

In [12], fuzzy based intelligent agent routing was established to find packet loss rate, optimal parameters, membership functions and repairing of path broken. The major limitation of this work is, reliability inclusion was avoided. In general, network performance depends on reliability of link as well as nodes. Link 
connectivity was also not maintained in this routing.

Multicast ad hoc on-demand distance vector routing protocol (MAODV) [13] is the multicast extension of the AODV, which is a hard state, and tree based protocol where group member joins/leaves the network and updates the request table through a Group Hello message. ADB presents an adaptive backbone infrastructure to integrate the effectiveness of the flooding scheme and the efficiency of tree-based scheme. ADB protocol is realized with neighbor discovery process, core selection process and core forwarding process to manage group level and backbone level operations for multicasting.

The Lantern-Tree-Based (LTB) in [14] is a bandwidth constrain QoS multicast routing protocol. A lantern is defined as one or more sub-paths with a total bandwidth between a pair of two neighboring nodes. A lantern path is a path with one or more lanterns between a source and a destination. The multicast tree contains at least one lantern path between any of its source-destination pairs. Lantern tree protocol measures the bandwidth as the available amount of free slots based on CDMA-over-TDMA channel model at MAC layer. One drawback of LTB is the long time needed to find all the paths and to share and schedule the time slots. Another drawback is the use of high number of links, which increase the contention at the MAC layer.

QoS Multicast Routing Protocol (QMR) [15] is a hybrid scheme for supporting QoS routing. It is an on-demand mesh protocol connects group members using QoS paths. QMR define forwarding nodes that provide at least one path from each source to each destination. CDMA-over-TDMA is used to estimate the available bandwidth. A distributed admission control is used to enable intermediate nodes to reject the routes that not satisfy QoS requirement. The forwarding nodes are updated when multiple sources sending to the multicast group simultaneously. This prevents congestion and performs load balancing in the network.

The protocol, termed ODMRP (On-Demand Multicast Routing Protocol), is a mesh-based, rather than a conventional treebased, multicast scheme and uses a forwarding group concept (only a subset of nodes forwards the multicast packets via scoped flooding). It applies on-demand procedures to dynamically build routes and maintain multicast group membership. ODMRP is well suited for ad hoc wireless networks with mobile hosts where bandwidth is limited, topology changes frequently, and power is constrained [16].

In [17] presents an Entropy-based long-life multicast routing protocol in MAODV (E-MAODV). It uses entropy concepts to develop an analytical modeling, and selects the longlife multicast routing according to entropy metric. This improvement reduces the number of route reconstruction and ensures the route stability in dynamic mobile networks, but it increases complexity of route establishments. Furthermore, this paper takes no extra measure to repair broken tree branches. NMP-MAODV [18] (Node Mobility Prediction-MAODV) ensures non-disconnection communication by active-link switch before mobile node breaks away from upstream node's signal range. The multicast group members set the unified cycle and calculate the predicted departure time. If the result is less than threshold, then turn to the active-link switch process. If the link switch process fails, it will initiate the MAODV repair process. The improved protocol can operate properly in highly mobile network, but it increases additional control overhead due to On-demand QoS multicasting protocol is proposed in [19]-[21]. This protocol simultaneously use multiple paths or trees in parallel to meet the required bandwidth of a single QoS request within a delay bound between the source and the destination. The bandwidth is considered as the number of free slots using CDMA-over-TDMA channel model. They propose three multiple path construction strategies to enable the source node to aggregate the bandwidth over the links. The source computes the optimal routes to the destinations and manages the group membership, which overload the source with extra processing overhead. Using flooding to discover the paths add the processing overhead for non-member nodes and waste the network resources.

The Ad hoc Multicast Routing protocol utilizing Increasing ID-numbers (AMRIS [22]) assigns an identifier to each node in a multicast session. A per-multicast session delivery tree rooted at a special node (by 
necessity a sender) in the session joins all the group members. Assigning identifiers in increasing order from the tree root outward to the other group members maintain the tree structure. Election algorithms (not yet specified) may be required to choose one special node if multiple senders are present in a multicast session. All nodes in the network are required to support AMRIS and any node can be on a tree. All nodes are required to process the tree setup and maintenance messages that are transmitted by the root periodically. No global state is required to be maintained by nodes on the tree, and repairs to damaged links are performed locally. AMRIS currently assumes the existence of bidirectional links between network nodes. It also assumes that applications (multicast sessions) are long-lived, and hence, sacrifices route discovery latency to route recovery latency.

\section{The Proposed Approach (AIS-DSR)}

In this section, we design a RM-Approach multicast routing protocol by jointly employing the fuzzy logic system.Helpful Hints

\subsection{Proposed Fuzzy-Based Multicast Routing Protocol}

In order to calculate the lifetime of the route in the proposed method, we use different criteria under fuzzy environment. The desired criteria can be selected based on evaluating the route characteristics including the hop count, the control packets and the lowest energy consumption of the route node. Through the definition of the fuzzy linguistic rules, membership functions equal to each element in the linguistic set of the criteria for the hop count, the control packets, in the proposed method RM-Approach, the minimum energy and lifetime are defined. Using these criteria and the fuzzy logic, we provide a method to design membership functions. We assess ranking of criteria based on linguistic expressions such as very low (VL), Low (L), Medium (M), high (H) and very high (VH). The used rating scale Hop Count is indicated in Fig. 2, and the membership functions of control packets for five language values are indicated in Fig. 3, the used rating scale energy is indicated in Fig. 4.

\subsection{Evaluation of the Criteria}

Hop Count: we consider the hop count as an evaluation criterion of the lifetime of the route in the routing table. The hop count is the number of nodes along a route between the source and destination. Hop count in a route has a negative relationship with lifetime of the route, when the hop count is high, the probability of route failure is high due to the movement of nodes, therefore, the lifetime of the route will be less. So, when a route is in the routing table, the hop count will be checked regarding the criteria. As a result, the rules should be as follows:

H 1: If the hop count is very high, then the lifetime of the route is very low.

H 2: If the hop count is high, then the lifetime of the route is low.

H 3: If the hop count is average, then the lifetime of the route is average.

H 4: If the hop count is low, then the lifetime of the route is high.

H 5: If the hop count is very low, then the lifetime of the route is very high. 
Membership functions to rank the lifetime of the route and the hop count with the dimensionless index at $[0,1]$ are presented in Fig. 3, As we stated, the lifetime of the route has a generally negative relationship with the hop count.

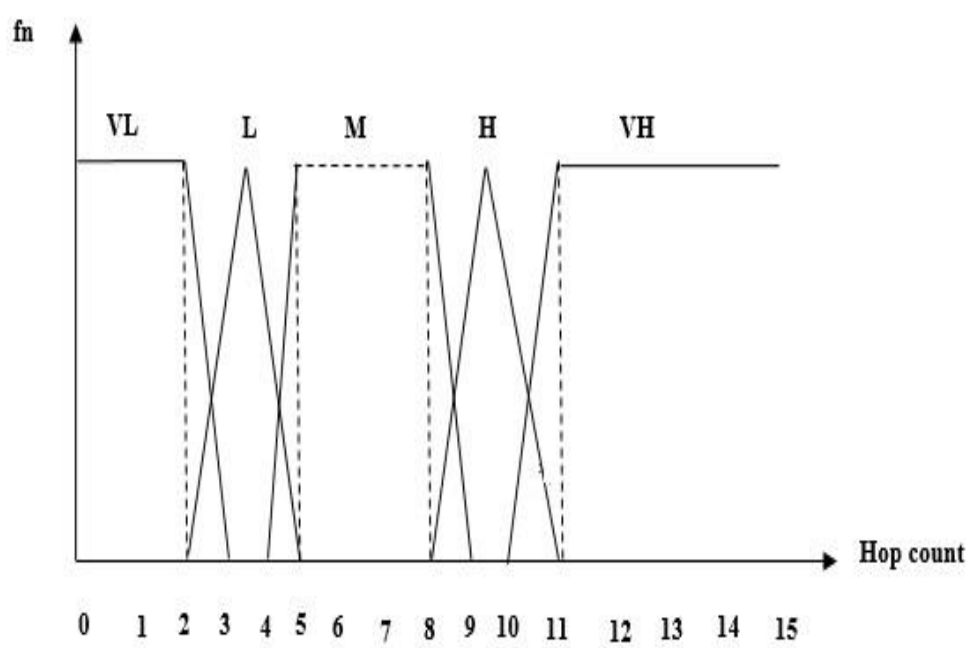

Fig. 3. Ranking scale for evaluation of the hop count parameter [VL (very low), L (low), M (medium), H

(high), VH (very high)].

Packet control: The packet control is the total number of packets sent and number of packets received. It is also a valid criterion for evaluating the lifetime of the route in the routing table. If the transfer is interrupted or the nodes have high mobility, the link may fail and the number of control packets increase. When the route becomes crowded, the shared bandwidth of the channels reduces, so that the route becomes unstable due to the retransmission of lost packets. Control packets can be of the following types: HELLO, RREQ RREP, RERR and RREP-ACK packets. If the number of the control packets sent by the interrupted nodes is high, it indicates that the route is probably unstable. Therefore, the high number of these packets means the potential loss of some links or the current packets. The rules of the fuzzy relationship between the number of control packets and the lifetime in the proposed method are as follows:

Pc 1: If the number of control packets is very high, then the
lifetime is very low.
Pc 2: If the number of control packets is high, then the
lifetime of the route is low.
Pc 3: If the number of control packets is average, then the
lifetime of the route is average.
Pc 4: If the number of control packets is low, then the lifetime
of the route is high.
Pc 5: If the number of control packets is very low, then the
lifetime of the route is very high.

Similarly, the rating of the number of control packets is stated by the dimensionless index of $[0,1]$ and the 
lifetime of the route has a negative relationship with the number of control packets. In addition, the energy characteristics can also be considered as an evaluation criterion. Fig. 4, as we stated, the number of control packets route has a generally negative relationship with the control packets.

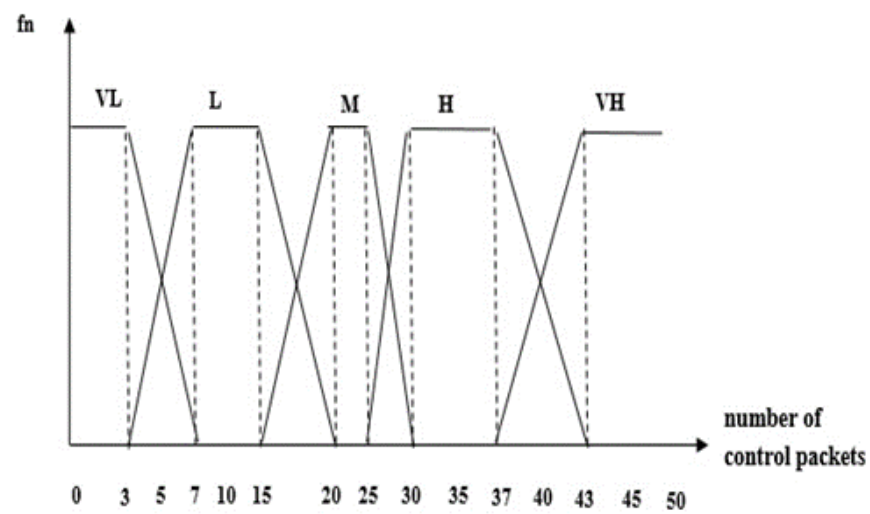

Fig. 4. Ranking scale for evaluation of the control packets parameter [VL (very low), L (low), M (middle), H (high), VH (very high)].

Minimum energy: if the energy of nodes is less, the route lifetime will be lower and the sooner it disappears. In the proposed method, energy consumption is used as an optional parameter to describe the energy condition of a node i. Energy consumption rate is shown as $E C_{i}$. The value of $E C_{i}$ is evaluated with a very simple method. As the energy consumption of a node is due to packet transfer activities, $E C_{i}$ is defined by the Equation (1):

$$
E C_{i}=\frac{\left(W_{r} * M_{r}+W_{s} * M_{s}+W_{o} * M_{o}\right)}{T}
$$

where $W_{r}, W_{s}$ and Wo are power consumed by the network interface when node i, sends, receives and overhears the packets; $M_{r}, M_{s}$ and $M_{o}$ are respectively the values of three types of packages; $T$ is the period in which the nodes i consumes its energy. Therefore, $E C_{i}$ is the average power consumption rate of node $\mathrm{i}$. Therefore, the time remaining for energy consumption by node $\mathrm{i}$, TR $\mathrm{i}$ is calculated by the Equation (2):

$$
T R_{i}=\frac{\left(R P_{i}\right)}{E C_{i}}
$$

where $R P_{i}$ is the remaining battery power node i. $T R_{i}$ is related to life route for all nodes in the route. This means that if the energy of each node is completed in the route, then the failure occurs. So $T R_{i}$ shows the remaining time of node $i$. Therefore, life route is associated with minimum of $T R_{i}$, which means that. If E-Min is low, the probability of the link failure will be high. Fig. 5 , as we stated, the energy route has a generally negative relationship with the energy route.

The fuzzy rules relationship between E-Min in the proposed approach will be as follows:

E 1: If E-Min is very high, then the route lifetime is very high.

E 2: If E-Min is high, then the route lifetime is high.

E 3: If E-Min is average, then the route lifetime is average.

E 4: If E-Min is low, so route lifetime is low.

E 5: If E-Min is very low, then the route lifetime is very low 


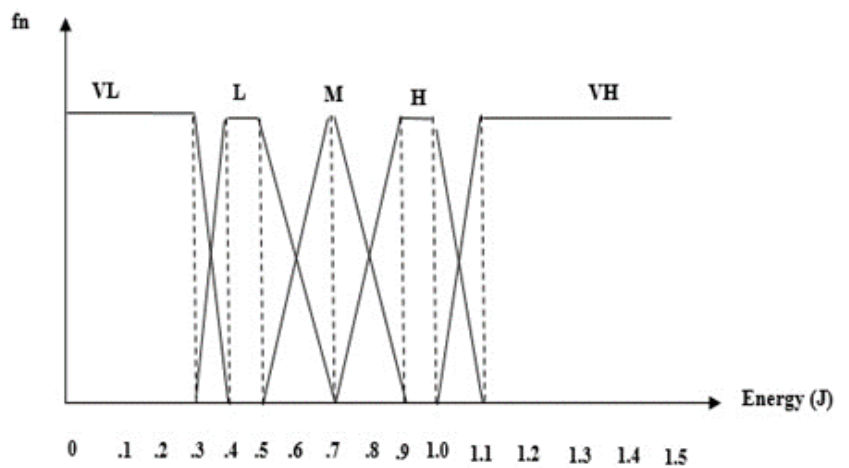

Fig. 5. Ranking scale for evaluation of the minimum energy parameter [VL (very low), L (low), M (middle), H (high), VH (very high)].

Energy minimum (E-Min), unlike the number of steps and the number of control packets, has a direct relationship with route lifetime. According to the first two criteria, the membership function can be considered as an indicator to measure E-Min between [0,1]. According to this concept, the range of energy reserve, $E-\min$ is defined by the Equation (3) and $R z E-\min$ is defined by the Equation (4):

$$
\begin{gathered}
E-\min =\min (T R i) \\
R z E-\min =(1-(E-\min ))
\end{gathered}
$$

Ranking of energy parameters is combined with the previous two rating criteria (the number of steps and the number of control packets) and has created a set of route rankings. The three rankings should be weighted to get the final ranking. In general, to prevent any final amount after the transfer, a practical value which is much lower or higher than the equivalent boundary must be assigned as a rating of very low or high. The above rules can be combined with a rule base such as base provided in Table 1. This rule base is used for fuzzy system which is described below.

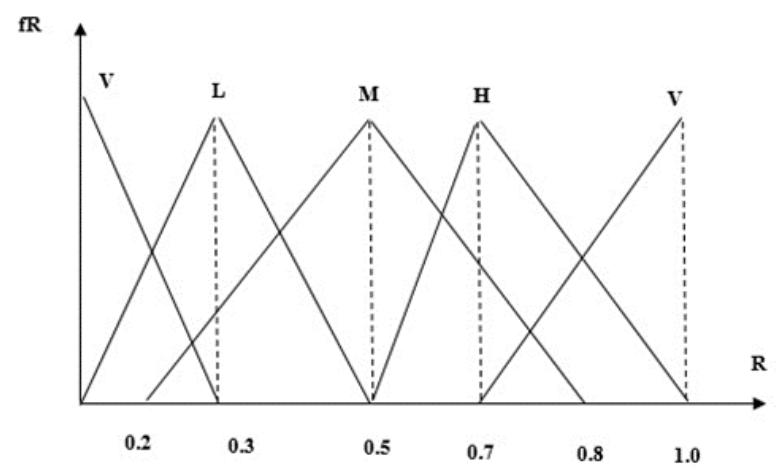

Fig. 6. The membership functions for language ranking values $\quad[\mathrm{VL}(0,0,0,0.3), \mathrm{L}(0,0.3,0.3,0.5), \mathrm{M}$ $(0.2,0.5,0.5,0.7), \mathrm{H}(0.5,0.7,0.7,1), \mathrm{VH}(0.7,1,1,1)]$.

\subsection{Using Fuzzy System}

Fuzzy system describes the uncertain and unknown phenomenon. The fuzzy system is a knowledge or rule -based system. The main part of the knowledge base contains if - then rules. The front lines include the rules of fuzzy variables that are produced using input and the consequent contains the fuzzy system output. The proposed method evaluated different routes in lifetime rankings to equal destinations under several weighted criteria. The membership functions' values are assigned to route $\mathrm{i}$ as $F_{i}$. Also we equal 
membership functions of $H_{i}, P C_{i}, E_{i}$ and RzE (i) with the number of step, control package, E-Min and $R z E-\min$. Membership functions of $\mathrm{E}(i)$ and $R z E$ (i) for the amount of the rankings $E-\min$ are expressed as below:

Table 1. Ranking of Alternate Routes Based on Three Expressed Criteria

\begin{tabular}{|c|c|c|c|}
\hline Route & Hop Count & $\begin{array}{l}\text { Control } \\
\text { packets }\end{array}$ & $\begin{array}{c}\text { Minimum energy } \\
\text { (E-Min) }\end{array}$ \\
\hline$R 1$ & $V H$ & $H$ & $L$ \\
$R 2$ & $M$ & $V L$ & $M$ \\
$R 3$ & $H$ & $L$ & $V H$ \\
$R 4$ & $L$ & $M$ & $H$ \\
$R 5$ & $V L$ & $V H$ & $L$ \\
\hline
\end{tabular}

The final ranking of fuzzy fitness index is for each candidate route 1 is calculated using the following Equation (5) and Equation (6)::

$$
\begin{gathered}
E(i)=(\text { a.b.c.d }) \\
R z E(i)=(1-d .1-c .1-b .1-a)
\end{gathered}
$$

\subsection{The Proposed Method Steps}

Multicast sending from a source to multiple destinations, each destination has received RREQ from source and sends RREPs to the source. In the proposed method, we only look at multicast source to a destination via multiple routes, which is as follows:

The first phase of route selection: First, the source node sends the RREQs to the multicast group. Recipients sent again unique RREQs in the form of a flood and then destination responded to the origin via RREP packets and the number-step, the remaining power and sent control packages have recorded intermediate nodes. Suppose that the return routes from a destination are $R 1, R 2, R 3, R 4$ and $R 5$. As a result, the origin will have three criteria-based assessments as step number, Control package numbers and E-Min number created from different routes. The legal bases are used which are listed in Table 2.

The second phase of the appointment of membership functions and the number of control packets: $H(i), \mathrm{PC}(i)$, are the membership functions of the ranking amounts of the number of steps and the number of control packets, $H(i)$, and $P C(i)$, are appointing to any route candidate $i$.

The third phase of the appointment of $E$ (i) and $R z E$ (i) membership functions: Membership functions of ranking amounts of E-Min and the ranking amount of RzE-Min, $E(i)$ and $R z E(i)$ are respectively appointed to any route candidate $i$.

The fourth phase of fitness index: Weight values of $W_{H}, W_{P C}$ and $W_{R Z E}$ are determined, then we deal with the calculation of combined fitness indexes, $F(i)$. Using the combinational rules database to obtain the final Fuzzy Ranking Index, which is displayed as $C F(i)$, the fuzzy fitness indexes of $R L P(i)$, are calculated for the lifetime of all routes. The source of the two stable separate rotes are selected from among the candidate routes. The selected optimal routes should have a higher lifetime in the routing table.

\section{Performance Evaluation}

In this part, we evaluate the performance of the proposed method (RM-Approach) in the problem of multicast routing.

\subsection{Performance Metrics}


We conduct extensive simulations to evaluate the effectiveness and performance of our RM-Approach and compare it with RSGM approach [23]-[25]. We evaluate the packet delivery ratio, end-to-end delay and lifetime.

\subsubsection{Packet delivery rate (PDR)}

PDR equals the number of successfully delivered data packets delivered to destination nodes to the total number of transmitted data packets from the source node [26]-[28]. Thus, we can define PDR as shown in Equation (7).

\subsubsection{End to end delay}

$$
\left(\frac{\sum_{\mathrm{j}=1}^{\mathrm{n}} \text { Packets recieved }}{\sum_{\mathrm{j}=1}^{\mathrm{n}} \text { Packets originated }}\right)
$$

This is the average delay between the sending of packets by the source and its receipt by the receiver. This includes all possible delays caused during data acquisition, route discovery, queuing, processing at intermediate nodes, retransmission delays at the MAC, propagation time, etc. It is measured in milliseconds. The lower value of end-to-end delay means the better performance of the protocol. The end-to-end delay is calculated in Equation (8) follows: [28]-[30].

$$
\left(\frac{\sum_{\mathrm{j}=1}^{\mathrm{n}} \text { Delivery Time }-\sum_{\mathrm{j}=1}^{\mathrm{n}} \text { Arrival Time }}{\sum_{\mathrm{j}=1}^{\mathrm{n}} \text { Recieved packets }}\right)
$$

\subsubsection{Network lifetime}

Node remaining energy is one of major issues in mobile ad-hoc networks presented here. As stated, consumed energy level directly influences network lifetime; therefore, network lifetime increases using high-energy routes.

\subsection{Simulation Results and Analysis}

We have implemented RM-Approach in the NS-2 on Fedora 10. The simulation parameters are given in Table 1.

Table 2. Simulation Parameters

\begin{tabular}{|l|c|}
\hline Parameters & Value \\
\hline Channel Type & Channel/Wireless channel \\
\hline Publication Type & Two ray ground \\
\hline Network Interface & Wireless Phy \\
\hline Antenna & Omni Antenna \\
\hline Simulation Area $(\mathrm{m} \times \mathrm{m})$ & $1000 \times 1000$ \\
\hline MAC layer & MAC/802.11 \\
\hline Traffic Type & CBR \\
\hline Queue Type & Drop Tail \\
\hline Number of nodes & 60 \\
\hline Primary energy & 10 Jules \\
\hline Mobility speed & $10,20,30,40,50$ and 60 \\
\hline Time simulation & $200 \mathrm{~s}$ \\
\hline
\end{tabular}


Fig. 6 shows the result of mobility vs packet delivery ratio. from the results, our proposed scheme achieves high packet delivery ratio than the existing schemes because of stability. deployed in the reliable multicast routing. Packet arrived at the destination node is more. Reliable multicast backbone ensures that packets are travelled through reliable links. Link between the nodes are well connected together to avoid congestion. In previous schemes, there was no stable routing deployed and the consumption of query packets is more. It will lead to high packet flooding. In the proposed protocol, first fuzzy adaptive routing ensures reliability of node and link. After that packets will be initiated for transmission.

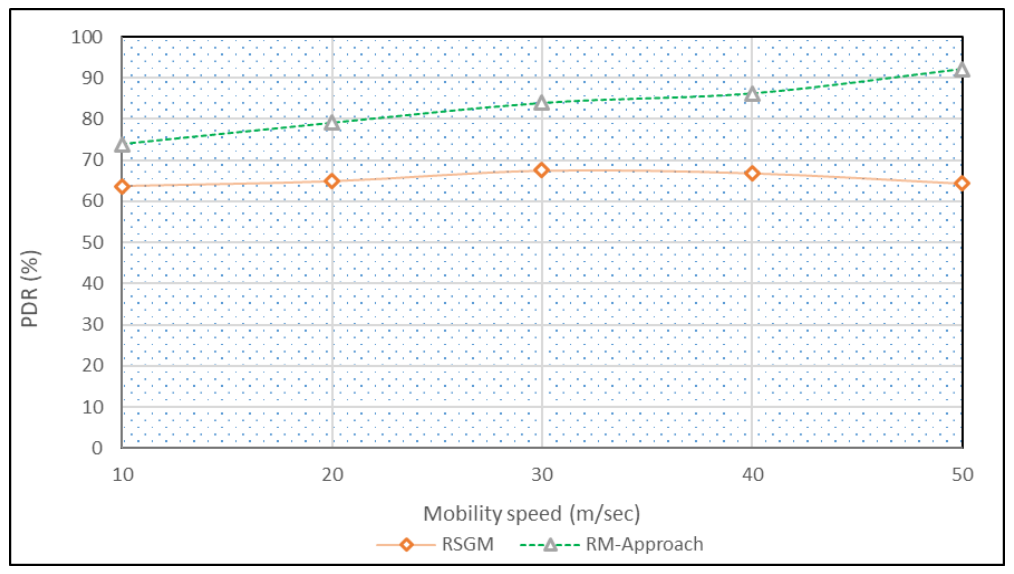

Fig. 6. PDR vs. mobility speed.

In Fig. 7, speed is varied as 10, 20...50. When we increase the speed, the mobility is also getting increasing. Mobility of nodes will lead to network partition. In our proposed model, mobility is kept less dynamic. Nodes transmit the packet towards the destination with less delay. Packets propagating delay and transmission delay are kept low. The proposed protocol has low end-to-end delay per packet than the existing routing schemes.

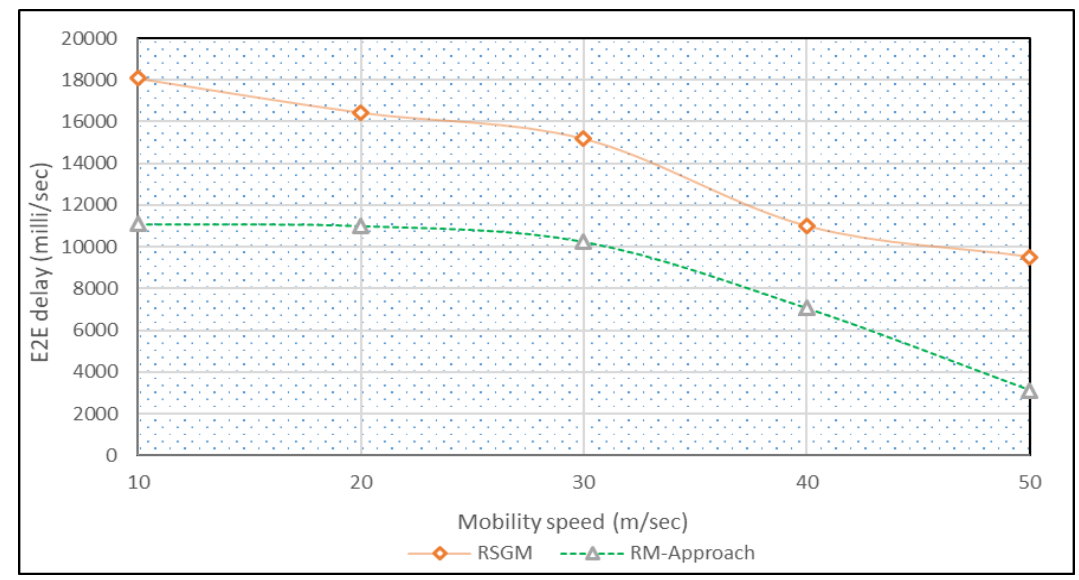

Fig. 7. End to end delay vs. mobility speed.

Node remaining energy is one of major issues in mobile ad-hoc networks presented here. As stated, consumed energy level directly influences network lifetime; therefore, network lifetime increases using high-energy routes. Fig. 8 shows that suggested protocol (RM-Approach) outperforms other protocol in network lifetime indicating supremacy of this protocol in energy usage and increased network lifetime. 


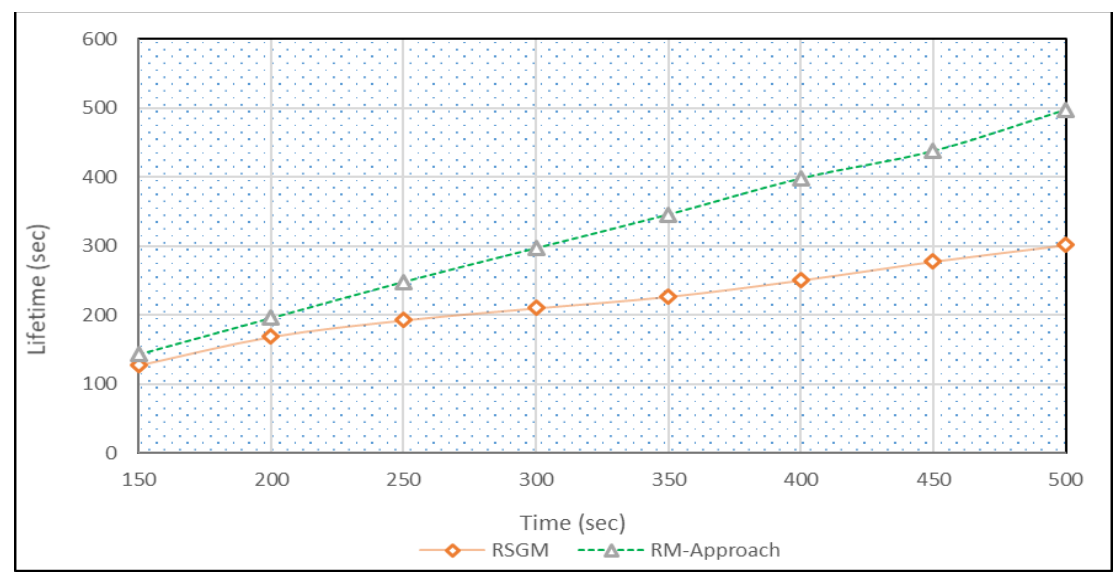

Fig. 8. Lifetime vs time.

\section{Conclusion}

In this paper, we proposed a reliable based multicast routing (RM-Approach) to select two comparably stable routes by computing dynamic route lifetime for multicast routing on multicasting for mobile ad-hoc networks (MANETs). The model compares and ranks different route lifetimes by the weighted multi-criteria, which are based on number of hop count, number of control packets and minimum consumption energy. The simulation results of the packet delivery ratio, lifetime and average end-to-end delay are evaluated with respect to mobility speed that illustrates the effectiveness of the proposed protocol compared to existing protocols such as RSGM. In our future work, we plan to devise a mechanism to secure MANETs against the malicious attack and test it intensively under different network conditions.

\section{References}

[1] Jamali, S., \& Fotohi, R. (2016). Defending against wormhole attack in manet using an artificial immune system. New Review of Information Networking, 21(2), 79-100.

[2] Sarkohaki, F., Fotohi, R., \& Ashrafian, V. (2017). An efficient routing protocol in mobile ad-hoc networks by using artificial immune system. International Journal of Advanced Computer Science and Applications(IJACSA), 8(4).

[3] Fotohi, R., Yaser E., \& Mohammad, S. (2016). A new approach for improvement security against DoS attacks in vehicular Ad-hoc network. International Journal of Advanced Computer Science and Applications, 7(7), 10-16.

[4] Zandiyan, S., Reza, F., \& Marzieh, K. (2016). P-method: Improving AODV routing protocol for against network layer attacks in mobile Ad-Hoc networks. International Journal of Computer Science and Information Security, 14(6), 95.

[5] Behzad, S., Reza, F., \& Fathulah, D. (2015). Defense against the attacks of the black hole, gray hole and wormhole in MANETs based on RTT and PFT. International Journal of Computer Science and Network Solutions (IJCSNS), 3(3), 89-103.

[6] Sarkohaki, F. (2012). A simulative comparison of DSDV and OLSR routing protocols. Australian Journal of Basic and Applied Sciences, 6(12), 373-378.

[7] Jamali, S., Fateme S., \& Reza, F. (2013). Providing a fault-tolerant algorithm for on-chip interconnection networks. International Research Journal of Applied and Basic Sciences (IRJABS), 4(7), 1700-1705.

[8] Gossain, H. (2004). Supporting MAC layer multicast in IEEE 802.11 based MANETs: Issues and solutions. Proceedings of the 29th Annual IEEE International Conference on Local Computer Networks. 
[9] Yao, G., Bi, J., \& Vasilakos, A. V. (2015). Passive IP traceback: Disclosing the locations of IP spoofers from path backscatter. Information Forensics and Security, 10, 471-484.

[10] Kunz, T., \& Ed, C. (2001). Multicasting in ad-hoc networks: Comparing MAODV and ODMRP. Proceedings of the Workshop on Ad hoc Communications.

[11] Santhi, G., \& Alamelu, N. (2012). Fuzzy-cost based multiconstrained QoS routing with mobility prediction in MANETs. Egyptian Informatics Journal, 13, 19-25.

[12] Budyal, V. R., \& Manvi, S. S. (2013). Intelligent agent based delay aware QoS unicast routing in mobile Ad hoc networks. International Journal of Multimedia and Ubiquitous Engineering, 8(1), 11-28.

[13] Awerbuch, B., Holmer, D., Nita-Rotaru, C., \& Rubens, H. (2002). An on-demand secure routing protocol resilient to byzantine failures. Proceedings of the 1st ACM Workshop on Wireless Security (pp. 21-30).

[14] Badis, H., Munaretto, A., Agha, K. A., \& Pujolle, G. (2003). QoS for ad hoc networking based on multiple metrics: bandwidth and delay. Proceedings of the 5th IEEE International Conference on Mobile and Wireless Communications Networks (pp. 15-18).

[15] Bao, C. W., \& Liao, W. (2005). Performance analysis of reliable MAC-layer multicast for IEEE 802.11 wireless LANs. Proceedings of 2005 IEEE International Conference on Communications.

[16] Barakovic, S., Kasapovic, S., \& Barakovic, J. (2010). Comparison of MANETS routing protocols in different traffic and mobility models. Telfor Journal, 2(1).

[17] Boukerche, A., Turgut, B., Aydin, N., Ahmad, M. Z., Bölöni, L., \& Turgut, D. (2011). Routing protocols in ad hoc networks: A survey. Computer Networks, 55(13), 3032-3080.

[18] Basagni, S., Conti, M., Giordano, S., \& Stojmenovic, I. (2004). Mobile ad hoc networking. John Wiley \& Sons.

[19] Chaturvedi, A. (2013). Route discovery protocol for optimizing the power consumption in wireless Ad-hoc network. International Journal of Advanced Computer Research, 3(1).

[20] Chen, C. W., \& Weng, C. C. (2012). A power efficiency routing and maintenance protocol in wireless multi-hop networks. Journal of Systems and Software, 85(1), 62-76.

[21] Chen, H. F. (2012). Stateless Multicast Protocol for Ad Hoc Networks.

[22] De, R. F., Guerriero, F., \& Fazio, P. (2012). Link-stability and energy aware routing protocol in distributed wireless networks. IEEE Transactions on Parallel and Distributed Systems, 23(4), 713-726.

[23] Jamali, S., \& Reza F. (2017). DAWA: Defending against wormhole attack in MANETs by using fuzzy logic and artificial immune system. The Journal of Supercomputing, 1-24.

[24] Fotohi, R. (2013). An improvement over AODV routing protocol by limiting visited hop count. International Journal of Information Technology and Computer Science (IJITCS), 5 (9), 87.

[25] Fotohi, R., Shahram J., \& Fateme, S. (2013). Performance evaluation of AODV, LHC-AODV, OLSR, UL-OLSR, DSDV routing protocols. International Journal of Information Technology and Computer Science (IJITCS), 5(10), 21.

[26] Fotohi, R., \& Shahram J. (2014). A comprehensive study on defence against wormhole attack methods in mobile Ad hoc networks. International Journal of Computer Science \& Network Solutions, 2(5), 37-56.

[27] Behzad, S., Reza, F., \& Mehdi, E. (2013). Queue based job scheduling algorithm for cloud computing. International Research Journal of Applied and Basic Sciences.

[28] Fotohi, R., \& Mehdi, E. (2013). A cluster based job scheduling algorithm for grid computing. International Journal of Information Technology and Computer Science (IJITCS), 5(12), 70.

[29] Behzad, S., Reza F., \& Shahram, J. (2013). Improvement over the OLSR routing protocol in mobile Ad Hoc networks by eliminating the unnecessary loops. International Journal of Information Technology and Computer Science (IJITCS), 5(6), 16.

[30] Reza, F., Mehdi, E., Fateme, S., Shahram, B., \& Jaber, H. (2012). An improvement over threads 
communications on multi-core processors. Australian Journal of Basic and Applied Sciences, 6(12), 379-384.

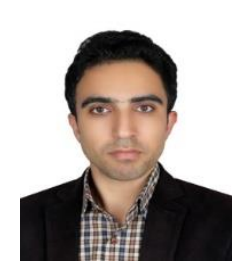

Shahram Zandian Taifeh Ajirloo was born in Ardabil, Iran, in 1988. He received the B.Sc degree in computer engineering from the University of Rasht, gilan, Iran, in 2011, and the M.Sc degrees in computer engineering from the Ardabil Science and Research Branch, Islamic Azad University, Ardabil, Iran, in 2017, respectively.

His current research interests include computer networks, mobile ad hoc network and internet of things. He worked as a software engineer for informatics services corporation.

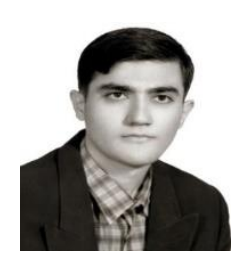

Abbas Mirzaei Somerin was born in Ardabil, Iran, in 1982. He received the M.Sc degree in computer engineering from the Islamic Azad University of Tabriz branch, East Azerbaijan, Iran, in 2007, and the Ph.D degrees in computer engineering from the Malek-Ashtar University of Technology, Tehran, Iran, in 2017, respectively.

His current research interests include computer networks, mobile ad hoc network and big data. He worked as a master for Islamic Azad University. 